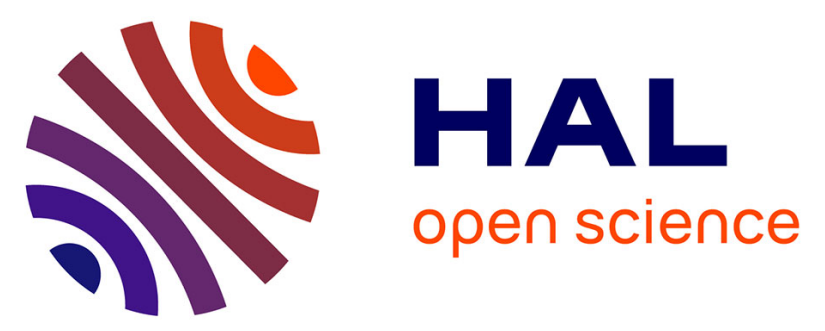

\title{
Apport des jaugeages ponctuels à la reconstitution de chroniques de débits moyens journaliers par simulation pluie-débit : l'exemple du bassin Rhin-Meuse
}

Julien Plasse, Gilles Drogue, Didier Francois, Philippe Battaglia, Céline Conan

\section{- To cite this version:}

Julien Plasse, Gilles Drogue, Didier Francois, Philippe Battaglia, Céline Conan. Apport des jaugeages ponctuels à la reconstitution de chroniques de débits moyens journaliers par simulation pluie-débit: l'exemple du bassin Rhin-Meuse. La Houille Blanche - Revue internationale de l'eau, 2014, 1, pp.45 52. $10.1051 / \mathrm{lhb} / 2014007$. hal-01697837

\section{HAL Id: hal-01697837 \\ https://hal.univ-lorraine.fr/hal-01697837}

Submitted on 23 Jun 2020

HAL is a multi-disciplinary open access archive for the deposit and dissemination of scientific research documents, whether they are published or not. The documents may come from teaching and research institutions in France or abroad, or from public or private research centers.
L'archive ouverte pluridisciplinaire HAL, est destinée au dépôt et à la diffusion de documents scientifiques de niveau recherche, publiés ou non, émanant des établissements d'enseignement et de recherche français ou étrangers, des laboratoires publics ou privés. 


\title{
Apport des jaugeages ponctuels à la reconstitution de chroniques de débits moyens journaliers par simulation pluie-débit : l'exemple du bassin Rhin-Meuse
}

\author{
Julien PLASSE${ }^{1}$, Gilles DROGUE${ }^{1}$, Didier FRANCOIS ${ }^{1}$, Philippe BATTAGLIA², Céline CONAN ${ }^{3}$
}

\author{
1. Université de Lorraine (EA LOTERR) - Île du Saulcy BP 3030957006 Metz cedex 1, France - gilles.drogue@univ-lorraine.fr \\ DREAL Lorraine - Rue Claude Chappe BP 9503857071 Metz, France - philippe.battaglia@developpement-durable.gouv.fr \\ 3. Agence de l'Eau Rhin-Meuse - Rue du ruisseau Rozérieulles BP 3001957161 Moulins Lès Metz, France - celine.conan@eau-rhin-meuse.fr
}

\begin{abstract}
RÉSUMÉ. - L'exploitation rétrospective des données physico-chimiques de qualité des eaux courantes requiert la connaissance du contexte hydrologique (chroniques de débits) dans lequel s'effectue le prélèvement d'eau. Cette information hydrologique n'est pas toujours connue au point de prélèvement ou de façon seulement discrète (débits ponctuels). Cet article présente les résultats d'une analyse comparative de méthodes de calcul des débits des cours d'eau par simulation pluie-débit applicables au droit des stations du réseau de contrôle de surveillance (RCS) gérées par l'Agence de l'Eau Rhin-Meuse. L'étude repose sur un réseau de mesure hydrométrique relativement dense et un patrimoine de données hydrologiques couvrant la période 1990-2009. Les résultats de l'étude montrent que la disponibilité d'une information hydrométrique ponctuelle même parcimonieuse au point de prélèvement, permet d'améliorer significativement la reconstitution des chroniques de débits journaliers et la définition du voisinage hydrologique utilisé lors de leur reconstitution. Tout suivi physico-chimique des eaux courantes devrait donc être doublé d'un suivi hydrométrique minimal, indépendant du suivi physico-chimique de manière à couvrir toute la gamme de débits de la rivière et permettant de reconstituer par modélisation hydrologique une chronique de débits aux points des réseaux RCS dépourvus de cette information à caractère opérationnel ou patrimonial.
\end{abstract}

Mots-clés : régionalisation, modélisation pluie-débit, débits ponctuels, bassin non jaugé, bassin Rhin-Meuse

\section{Using point flow measurements for guided reconstruction of daily streamflow time series through continuous flow simulation: a regional case study on the Rhine-Meuse district}

\begin{abstract}
Insufficient hydrological knowledge hampers the reanalysis of river quality measurements. For most of the pollution-control sites monitored by the French Water Agency, streamflow data are generally missing or are available only as few point gauging records. This paper presents a comparative study of methods of daily streamflow time series reconstruction based on rainfall-runoff modeling. Their performance is evaluated through data running from 1990 to 2009 over a rather dense streamflow monitoring network. Results show that a small number of point gauging measurements may help to significantly improve the reconstruction of daily streamflow data series for almost ungauged sites. Point gauging records are also useful to define the optimal neighborhood used during the regionalization process. At-site monitoring of chemical and physical status of surface water should additionally include a series of flow measurements well-distributed in time, especially in regions where hydrological data are scarce.
\end{abstract}

Key-words: regionalization, rainfall-runoff modeling, point flow measurement, ungauged basin, Rhine-Meuse district

\section{INTRODUCTION}

La Directive cadre sur l'eau (DCE) 2000/60/CE impose que soient établis des programmes de surveillance de l'état des eaux afin d'en dresser un tableau cohérent et complet au sein de chaque district hydrographique. Dans le cas des eaux de surface, les programmes portent notamment sur l'état écologique, l'état chimique et le potentiel écologique. Dans les bassins du Rhin et de la Meuse (partie française), les réseaux de contrôle de surveillance (RCS) sont composés de 107 sites pour le suivi des cours d'eau. Cependant, l'ensemble des réseaux mis en œuvre dans ces bassins regroupe 468 sites qui font l'objet de mesures régulières de la qualité des cours d'eau. Il est indispensable à l'Agence de l'Eau Rhin-Meuse (AERM) de connaître le contexte hydrologique de ces mesures pour mieux estimer et interpréter les données de flux de polluants et les valeurs d'indicateurs de pollution qui en sont dérivées. Le réseau hydrométrique régional, géré par les DREAL Alsace et Lorraine est composé actuellement de 188 stations permanentes de mesure des débits. Assez dense sur les grands cours d'eau, il l'est moins sur les bassins versants amont. Jusqu'ici, le contexte hydrologique sur les bassins versants amont et aux sites de mesure des RCS était le plus souvent documenté par des jaugeages ponctuels. Or cette information ponctuelle est insuffisante car elle ne permet pas de connaître la dynamique de l'évolution des débits pourtant nécessaire à la ré-analyse des observations issues du suivi physico-chimique des eaux de surface. Ainsi, si des chroniques de débits existent sur les grands cours d'eau et pour les sites de mesure des RCS implantés à proximité de stations hydrométriques, il convient de les reconstituer ailleurs, en l'absence de station hydrométrique. 
Le principal but de cet article est de comparer l'efficacité de différentes méthodes de reconstitution du débit en rivière en tout point du réseau hydrographique par simulation pluie-débit. Cette contribution méthodologique vise en outre à évaluer l'apport des jaugeages ponctuels (mesures ponctuelles du débit effectuées sur des points hydrographiques non instrumentés) pour la reconstitution de chroniques de débits moyens journaliers dans des zones hydrologiquement " pauvres » et en particulier au droit de sites de mesure des RCS qui ne sont pas proches d'une station hydrométrique.

\section{DONNEES ET METHODES}

\section{II.1. L'information hydrologique}

\section{II.1.1. Les chroniques de débits journaliers}

Les développements méthodologiques présentés ci-dessous se sont appuyés sur un réseau de mesure de 149 stations hydrométriques pérennes réparties sur un territoire d'environ $38000 \mathrm{~km}^{2}$ et offrant au moins 12 années de débits journaliers validées sur la période-cible 1990-2009. L'écoulement mesuré par ces stations retenues pour l'étude est jugé «naturel », c'est-à-dire peu ou pas influencé par les activités anthropiques (régulation, prélèvements, etc.). Ce lot de stations comprend 107 stations françaises situées dans les frontières hydrographiques de la partie française du bassin Rhin-Meuse ainsi que 42 stations situées sur leur pourtour, dans un corridor de 20 kilomètres, en France (29), en Allemagne (9) et en Belgique (4) (Figure 1). On dénombre 69 bassins de moins de $250 \mathrm{~km}^{2}$ (surfaces $\geq 5 \mathrm{~km}^{2}$ ), 41 dont la surface est comprise entre 250 et $800 \mathrm{~km}^{2}$ et 39 drainant plus de $800 \mathrm{~km}^{2}$ (surfaces $\leq 11500 \mathrm{~km}^{2}$ ). Les caractéristiques hydroclimatiques de la période d'observation et la longueur des chroniques garantissent la robustesse du protocole de test des méthodes d'estimation locale du débit.

\section{II.1.2. Les débits ponctuels}

Les données d'hydrométrie ponctuelle proviennent du SIERM (Système d'Information sur l'Eau Rhin-Meuse) géré par l'AERM et de la base BAREME qui contient les jaugeages de la DREAL Lorraine. Après pré-traitement de cette base de données, 14924 débits instantanés représentatifs d'un échantillon de 80 sites des RCS ont été extraits sur la période 1971-2010. On dénombre un minimum de 8 jaugeages par station avec une médiane et une moyenne respectivement de 142 et 187 jaugeages sur toute la période d'observation. Dans le cadre de cette étude, les données d'hydrométrie ponctuelle servent à juger du réalisme des débits ponctuels journaliers échantillonnés dans les chroniques de débits continues et utilisés lors des calculs de reconstitution des débits (voir II.4.2).

\section{II.2. La climatologie SAFRAN}

Les séries quotidiennes de pluie et d'ETP de bassin ont été déterminées pour les 149 bassins versants de notre échantillon à partir de la climatologie en points de grille SAFRAN validée sur la France (Quintana-Seguí et al., 2008). A une échelle plus fine que celle des zones Symposium (zones climatologiquement homogènes à des tranches d'altitude espacées de $300 \mathrm{~m}$ ), cette climatologie est affectée par des biais locaux se traduisant notamment, dans notre région d'étude, par un biais humide sur les Vosges du Nord et un biais sec sur les Vosges méridionales. Dans notre contexte de travail, ces biais n'ont pas fait l'objet d'une correction. En effet, il convient de rappeler qu'en modélisation pluie-débit, le calage des paramètres du modèle joue un double rôle i) l'un explicite

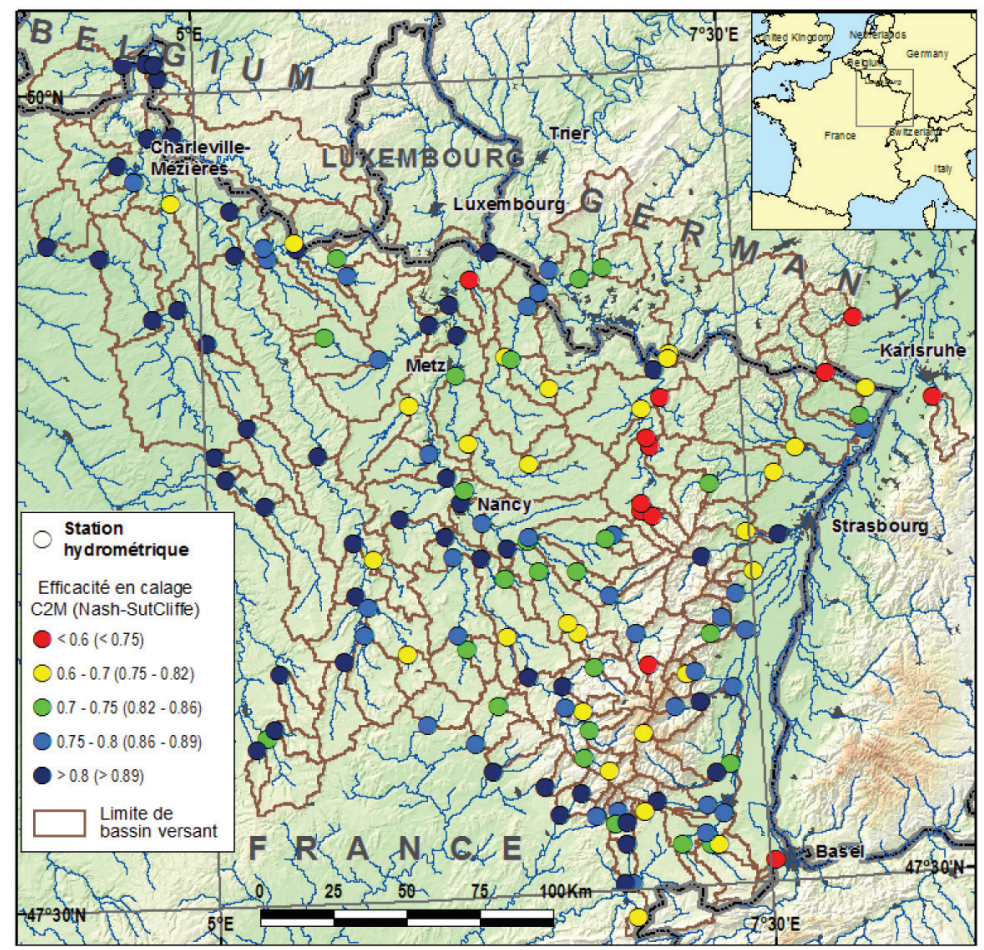

Figure 1 : Réseau de mesure hydrométrique et valeurs d'efficacité du modèle GR4J en calage au droit des 149 stations hydrométrique pérennes de l'étude. 
d'estimation des paramètres non connus ; ii) l'autre implicite de compensation sur ces seuls paramètres de toutes les erreurs liées au modèle et aux données. Ces biais peuvent néanmoins engendrer des vecteurs de paramètres atypiques susceptibles de limiter l'efficacité des méthodes de reconstitution du débit (voir III.3).

\section{II.3. Protocole de test}

\section{II.3.1. Présentation et calage/contrôle du modèle pluie-débit}

Les simulations pluie-débit ont été réalisées à l'aide du modèle hydrologique empirique global GR4J (Perrin et al., 2003). Celui-ci se caractérise par une structure à deux réservoirs (production et routage) et quatre paramètres libres à caler. Après une phase d'initialisation et de chauffe du modèle hydrologique d'une durée totale de 3 ans, l'efficacité du modèle GR4J a été évaluée pour chaque station hydrométrique à l'aide de la procédure de calage-contrôle croisée appliquée aux deux décennies de la période 1990-2009. La recherche du jeu optimal de paramètres a été effectuée à l'aide de l'algorithme BFGS (Byrd et al., 1995) par maximisation du coefficient de Nash-Sutcliffe (NS) calculé sur la racine carrée des débits journaliers afin d'accorder moins d'importance aux hautes eaux. Une fois le contrôle effectué, le modèle GR4J a été calé sur l'ensemble de la période de reconstitution (1990-2009) pour chacune des 149 stations disponibles ce qui a permis de constituer une bibliothèque régionale de paramètres. L'efficacité du modèle pluie-débit en calage (critère C2M défini par l'équation (1)) est spatialisée sur la Figure 1.

Le niveau d'efficacité est globalement satisfaisant. Une certaine disparité spatiale apparaît. L'absence d'un module neige dans notre modèle ainsi qu'un biais dans l'estimation des précipitations (cf. II.2) limitent les valeurs de C2M dans le massif vosgien. Le bassin de la Sarre concentre des performances particulièrement médiocres liées à des problèmes d'hydrométrie. D'autres valeurs d'efficacité faibles $(\mathrm{C} 2 \mathrm{M}<0.7)$ apparaissent ponctuellement et peuvent s'expliquer par des influences anthropiques non signalées dans la banque HYDRO (prélèvements, soutiens d'étiage, ...) ou des fonctionnements atypiques (ruisseau phréatique, karst, exsurgences et zones humides liées à l'aquifère des grès vosgiens, etc.). Notons que l'efficacité du modèle en calage ne rend pas compte des performances réelles du modèle hydrologique qui doivent être évaluées en contrôle.

\section{II.3.2. Validation croisée}

Afin d'évaluer la performance et le potentiel de généralisation des méthodes de reconstitution du débit, un protocole de validation croisée ( leave-one-out cross validation ») a été mis en œuvre. Chacune des 149 stations hydrométriques est tour à tour sortie de l'échantillon de stations et considérée comme un point-cible (point du réseau hydrographique où l'on souhaite connaître le débit journalier) non jaugé. Le débit reconstitué est ensuite comparé au débit observé à cette station. Un critère d'efficacité est ensuite calculé. Il s'agit dans notre cas du critère C2M (Nash-Sutcliffe borné entre -1 et 1) appliqué à la racine carrée des débits selon la formule (Mathevet et al., 2006):

$$
\mathrm{C} 2 \mathrm{M}_{\sqrt{\mathrm{Q}}}=\frac{N S_{\sqrt{\mathrm{Q}}}}{2-N S_{\sqrt{\mathrm{Q}}}}
$$

où $N S_{\sqrt{Q}}$ représente la critère d'efficacité de Nash-Sutcliffe comparant l'erreur quadratique moyenne du modèle calculé sur la racine des débits et la variance de la racine carrée des débits observés, Q le débit moyen journalier. Plus le critère $\mathrm{C} 2 \mathrm{M}$ est proche de 1 et meilleure est l'efficacité de la méthode de reconstitution.

\section{II.4. Reconstitution des chroniques de débit journalier}

Plusieurs méthodes sont testées ici, avec différentes façons d'exploiter l'information régionale et l'information locale disponibles. Les méthodes par voisinage géographique ont déjà fait l'objet d'une évaluation à l'échelle nationale (Oudin et al., 2008), mais seront donc analysées plus précisément ici sur le district Rhin-Meuse. L'approche d'exploitation des jaugeages ponctuels testée ici a été proposée par Rojas-Serna (2005) et est détaillée par Rojas-Serna et al. (soumis). D'autres travaux portant sur la prévision des débits sur des bassins non instrumentés (Seibert et Beven, 2009) ou le calage des modèles pluie-débit globaux (Perrin et al., 2007), ont mis en exergue la forte valeur informative d'un échantillon restreint de débits ponctuels.

\section{II.4.1. Reconstitution par voisinage géographique : méthode 1 (M1)}

La méthode M1 reconstitue le débit journalier au point-cible par transposition spatiale d'un ou plusieurs jeux de paramètres d'un modèle pluie-débit selon un critère de proximité spatiale entre les bassins-donneurs et le bassin-receveur contrôlé au point-cible. Chaque jeu de paramètres est ensuite transféré au point-cible où le modèle est utilisé en simulation avec les données d'entrée (pluie, ETP) du bassin contrôlé au point-cible. Le critère de proximité spatiale utilisé se définit comme une distance euclidienne entre centroïdes de bassins. Cette méthode n'exploite aucun débit ponctuel éventuellement disponible au point-cible. Des tests préliminaires ont révélé que l'efficacité de la méthode est meilleure lorsque l'hydrogramme journalier est reconstitué au point-cible en moyennant les hydrogrammes simulés avec chacun des vecteurs de paramètres des bassins-donneurs plutôt qu'en moyennant les vecteurs de paramètres des bassins-donneurs avant simulation. De plus, la méthode de reconstitution atteint son niveau de performance optimal avec quatre bassins-donneurs. L'estimation du débit moyen journalier au point-cible s'exprime alors comme suit :

$$
\overline{\hat{Q}}_{j}\left(f_{k}\right)=\frac{1}{4} \sum_{i=1}^{4} \hat{Q}_{j}\left(\theta_{i}, f_{k}\right)
$$

où $\overline{\hat{Q}}_{j}\left(f_{k}\right)$ représente le débit moyen journalier $(j)$ estimé au point-cible $k, f_{k}$ représente les vecteurs de forçage (pluie et ETP) du bassin dont $k$ définit l'exutoire, $\hat{Q}_{j}$ représente le débit journalier $(j)$ simulé à l'aide du vecteur de paramètres $\theta_{i}$ du i-ème bassin-donneur et des vecteurs de forçage $f_{k}$.

\section{II.4.2. Reconstitution par voisinage géographique et similitude hydrologique : méthode 2 (M2)}

La reconstitution du débit journalier s'opère à la fois par transposition spatiale des paramètres d'un modèle pluie-débit selon une stratégie de proximité spatiale (analogue à M1) et par similitude hydrologique. Ce modèle 
« hybride » suppose la détermination d'un rang composite pour chaque bassin-donneur potentiel. Ce rang composite est une combinaison linéaire pondérée telle que (Rojas Serna et al., soumis) :

$$
r_{j}=\alpha \times r_{\text {géo }}+(1-\alpha) \times r_{\text {hydro }}
$$

où $r_{j}$ est le rang composite du j-ème bassin-donneur, $r_{g e ́ o}$ le rang du j-ème bassin-donneur selon le critère de voisinage géographique (distance euclidienne entre centroïdes) et $r_{\text {hvdro }}$ le rang du j-ème bassin-donneur selon le critère de similitude hydrologique (Figure 2). Le coefficient de pondération $\alpha$ est à optimiser. Afin d'évaluer la sensibilité de cette méthode à la densité de l'information hydrométrique ponctuelle disponible au point-cible, nous avons fait varier la taille de l'échantillon de débits ponctuels sur la période cible ( $n$ variant de 1 à 200). La fréquence d'échantillonnage mensuelle des jaugeages ponctuels présents dans la BD SIERM étant uniforme, aucune contrainte n'a été appliquée à la probabilité de tirage.

Afin de retirer le biais temporel imputable au tirage aléatoire (risque de n'échantillonner que des bas débits) et obtenir des résultats d'une portée plus générale, le tirage aléatoire a été réitéré 100 fois pour chaque taille d'échantillon. Un critère $\mathrm{C} 2 \mathrm{M}$ moyen a ensuite été calculé sur la base de ces 100 itérations (Figure 2).

\section{II.4.3. Reconstitution par calage sur les débits échantillonnés au point-cible : méthode 3 (M3)}

La reconstitution du débit journalier s'effectue en 5 étapes : i) tirage aléatoire de $n$ valeurs de débits ponctuels au point-cible ( $n$ prend successivement les valeurs 30 , 50, 100 et 200) dans les chroniques de débits 1990-2009, ii) calage des paramètres du modèle GR4J au point-cible, iii) calcul du critère $\mathrm{C} 2 \mathrm{M}$, iv) pour des raisons de temps de calcul, les étapes i) à iii) ne sont réitérées que 20 fois, v) calcul d'un $\mathrm{C} 2 \mathrm{M}$ moyen. Pour ne pas biaiser la comparaison des performances entre méthodes, le $\mathrm{C} 2 \mathrm{M}$ est calculé sur l'ensemble des débits observés moins ceux ayant servi au calage du modèle GR4J.

\section{II.4.4. Reconstitution par transfert des paramètres du bassin-donneur le plus proche : méthode 4 (M4)}

Il s'agit d'une variante de la méthode M1 à un seul bassin-donneur. L'échantillon des 149 stations hydrométriques est complété à l'aide de stations présentant, une fois leurs séries de débits aboutées, entre 3 et 12 années de débits journaliers concomitants avec la période de reconstitution. Ceci porte l'effectif total de stations hydrométriques à 209. Cette méthode présente l'avantage d'exploiter les courtes chroniques de débits journaliers disponibles dans le proche voisinage du point-cible. La reconstitution du débit au point-cible résultera de la simulation d'un hydrogramme par GR4J en utilisant le jeu de paramètres calé

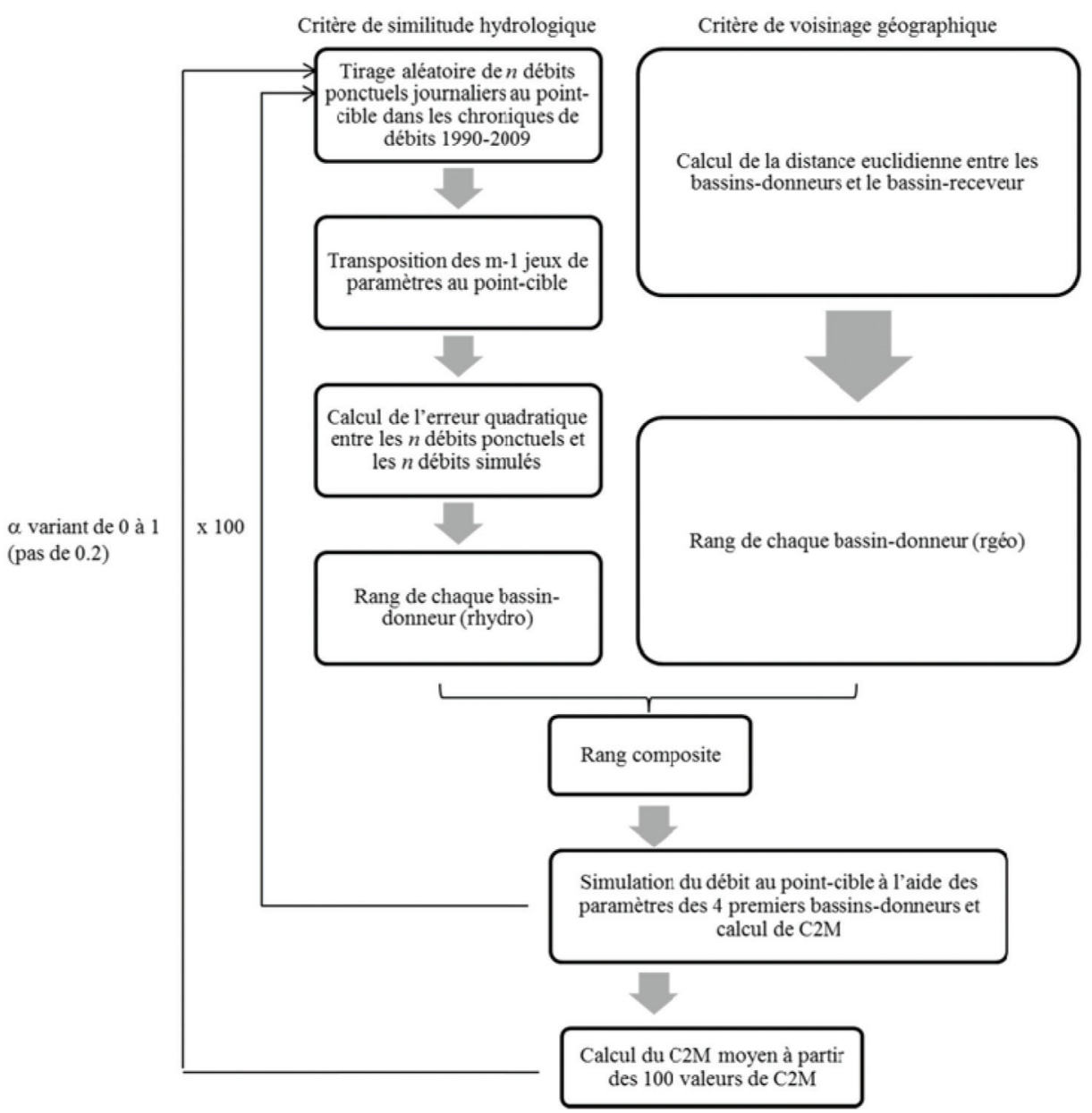

Figure 2 : Protocole de reconstitution de chroniques de débits journaliers par le modèle " hybride 》 (méthode M2) appliquée à chacune des 149 stations hydrométriques sur la période 1990-2009. 
sur le plus proche bassin-donneur toujours au sens de la distance euclidienne entre centroïdes de bassin. Notons que sur les 149 stations de l'échantillon de référence, 44 stations ont un premier voisin différent de celui identifié avec la méthode M1.

\section{RESULTATS ET DISCUSSION}

\section{III.1. Le modèle « hybride » (M2)}

Rappelons tout d'abord, en vertu de l'équation 3) que lorsque $\alpha=0$ (resp. $\alpha=1$ ), seul le critère de similitude hydrologique (resp. de proximité spatiale) intervient dans la définition du groupe de bassins-donneurs. La plus grande efficacité est obtenue avec 4 bassins-donneurs.

La Figure 3a met en évidence l'existence, pour chaque taille d'échantillon de débits ponctuels $(n)$, d'une valeur optimale (i.e. qui maximise le critère d'efficacité $\mathrm{C} 2 \mathrm{M}$ ) du coefficient $\alpha$. Elle montre qu'il suffit de disposer d'au moins 2 débits ponctuels pour améliorer la performance du modèle hybride, $\alpha$ prenant alors la valeur de 0.8 pour une valeur de $\mathrm{C} 2 \mathrm{M}$ de 0.65 . Par ailleurs si l'on dispose d'au moins 30 débits ponctuels, le poids de la composante géographique devient nul dans le modèle de régionalisation des débits (i.e. valeur maximale de $\mathrm{C} 2 \mathrm{M}$ pour $\alpha=0$ ).

Cet effet de seuil se retrouve sur la Figure $3 b$. Sur la gamme de 1 à 30 débits ponctuels, l'ajustement d'une relation mathématique de type linéaire (ou exponentiel sur toute la gamme de valeurs) entre le nombre de débits ponctuels échantillonnés et la valeur de $\alpha$ ouvre la perspective d'une prévision de la valeur d' $\alpha$ et donc du rang composite des bassins-donneurs pour un bassin réellement non jaugé.

\section{III.2. Calage sur les débits ponctuels échantillonnés (M3)}

L'intérêt de cette méthode n'est pas seulement d'étudier la stabilité et la robustesse du modèle de reconstitution selon le nombre de débits servant au calage du modèle pluie-débit mais également de savoir si une méthode naïve fondée sur un « simple » calage sur les débits ponctuels disponibles au point-cible est capable de rivaliser avec une méthode plus complexe (M1 ou M2).

Les résultats consignés dans le Tableau 1 confortent l'idée qu'il existe une corrélation positive entre la densité d'information hydrométrique disponible pour le calage du modèle hydrologique et son niveau de performance au point-cible en reconstitution. Ceci rejoint les conclusions de Perrin et al. (2007) qui constatent une meilleure capacité du modèle à identifier le comportement hydrologique d'un bassin versant lorsque l'information hydrométrique ponctuelle est plus riche en calage. Pour étayer ce point et juger de la représentativité des débits ponctuels échantillonnés par rapport aux débits instantanés issus des BD SIERM et BAREME, des quantiles caractéristiques de la distribution statistique des débits ont été comparés pour 15 stations des RCS disposant conjointement d'une information hydrométrique pérenne et ponctuelle (Figure 4). Malgré une certaine dispersion des points, les valeurs de quantiles de débit sont généralement proches. L'intervalle inter-décile (Q90-Q10) est du même ordre de
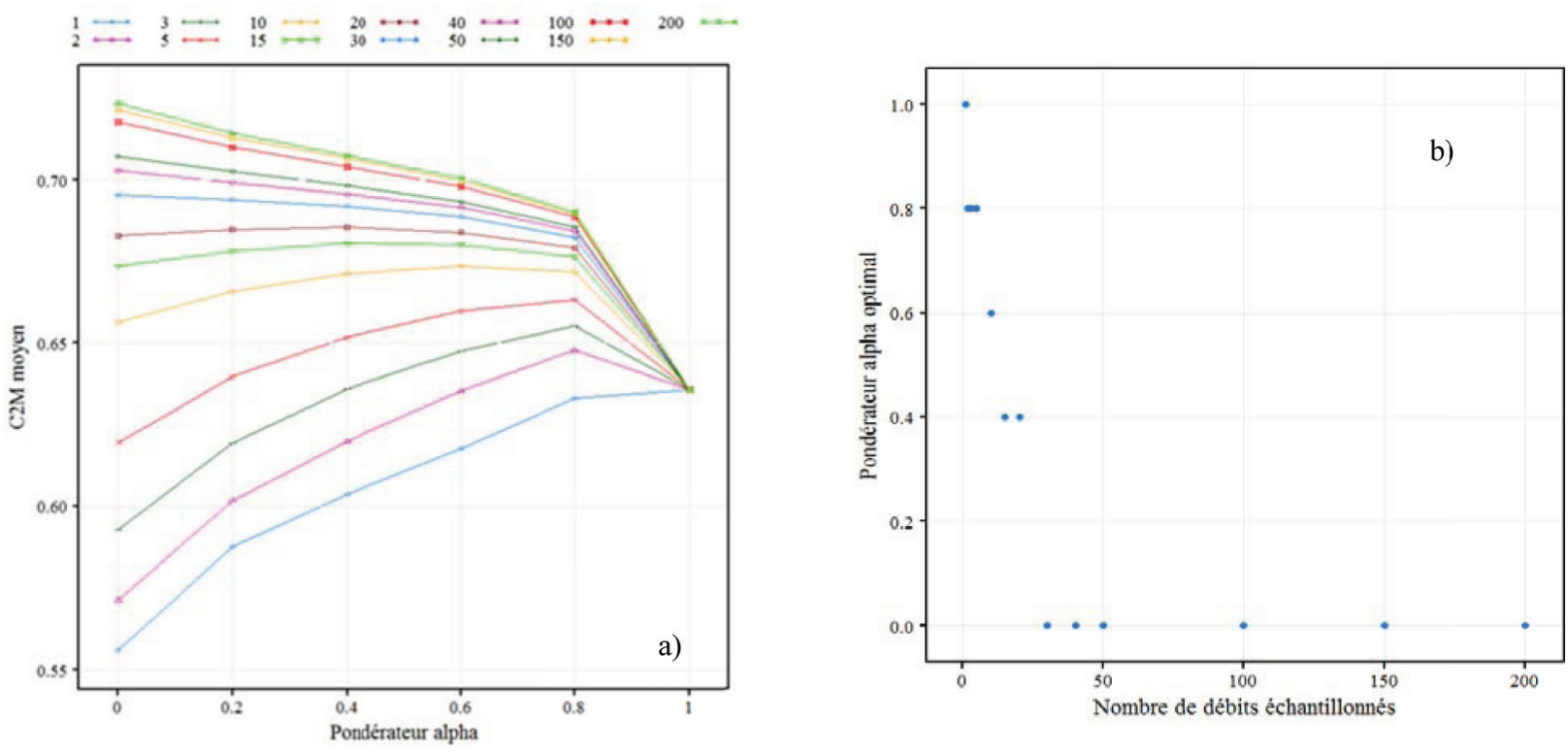

Figure 3 : (a) Evolution du critère C2M moyen (100 simulations, 149 stations) en fonction du coefficient de pondération a et du nombre $n$ (1 à 200) de débits ponctuels échantillonnés. (b) Relation entre le nombre $n$ de débits ponctuels échantillonnés et le coefficient de pondération a optimal.

Tableau 1 : Valeurs du critère C2M moyen et de l'écart-type du C2M (149 stations, 20 simulations).

\begin{tabular}{|l|c|c|c|c|}
\hline \multicolumn{1}{|c|}{ Nombre de débits ponctuels journaliers } & $\mathbf{3 0}$ & $\mathbf{5 0}$ & $\mathbf{1 0 0}$ & $\mathbf{2 0 0}$ \\
\hline C2M moyen & 0.65 & 0.68 & 0.71 & 0.72 \\
\hline Ecart-type du C2M & 0.08 & 0.06 & 0.04 & 0.03 \\
\hline
\end{tabular}



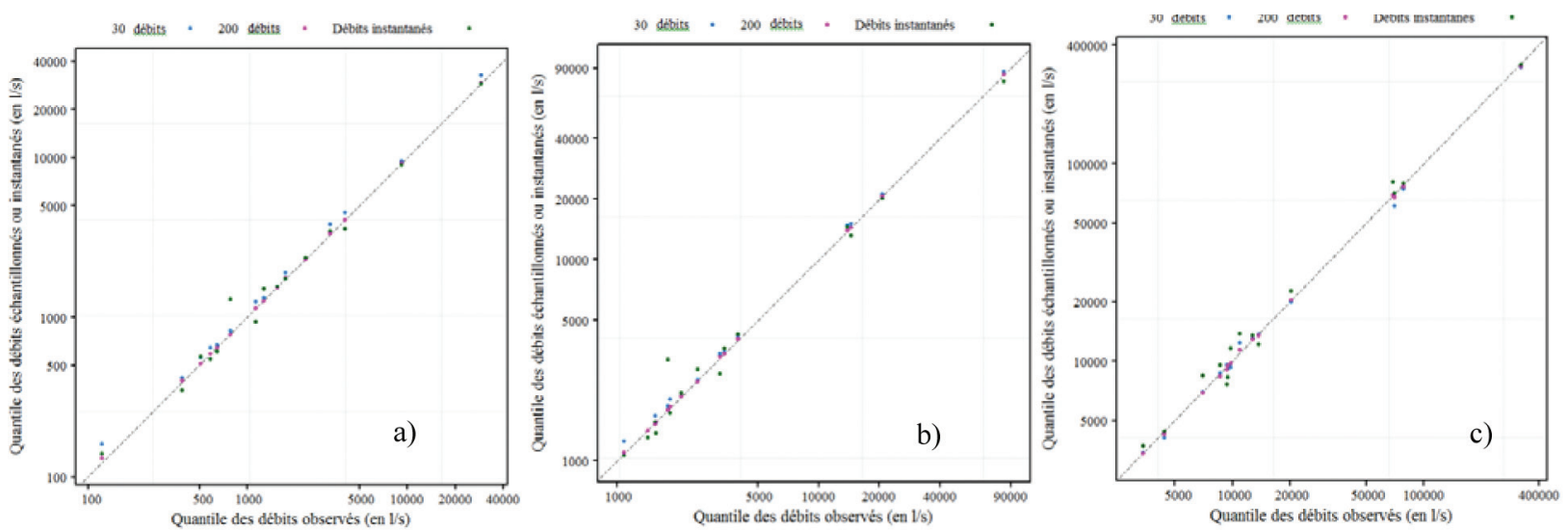

Figure 4 : Comparaison des quantiles de débits (échelle logarithmique) calculés sur les chroniques de débits journaliers observés, les séries de débits ponctuels échantillonnés $(n=30$ et $n=200)$ et les débits instantanés pour 15 stations des RCS très proches ou confondues avec une station hydrométrique (surfaces de bassin comprises entre 94 et $11500 \mathrm{~km}^{2}$; moyenne de 120 débits instantanés par station). a) Q10 (1 er décile) ; b) Q50 (médiane) ; c) Q90 (9e décile). Pour $n=30$ et $n=200$ les valeurs de quantiles correspondent à la moyenne des 100 tirages.
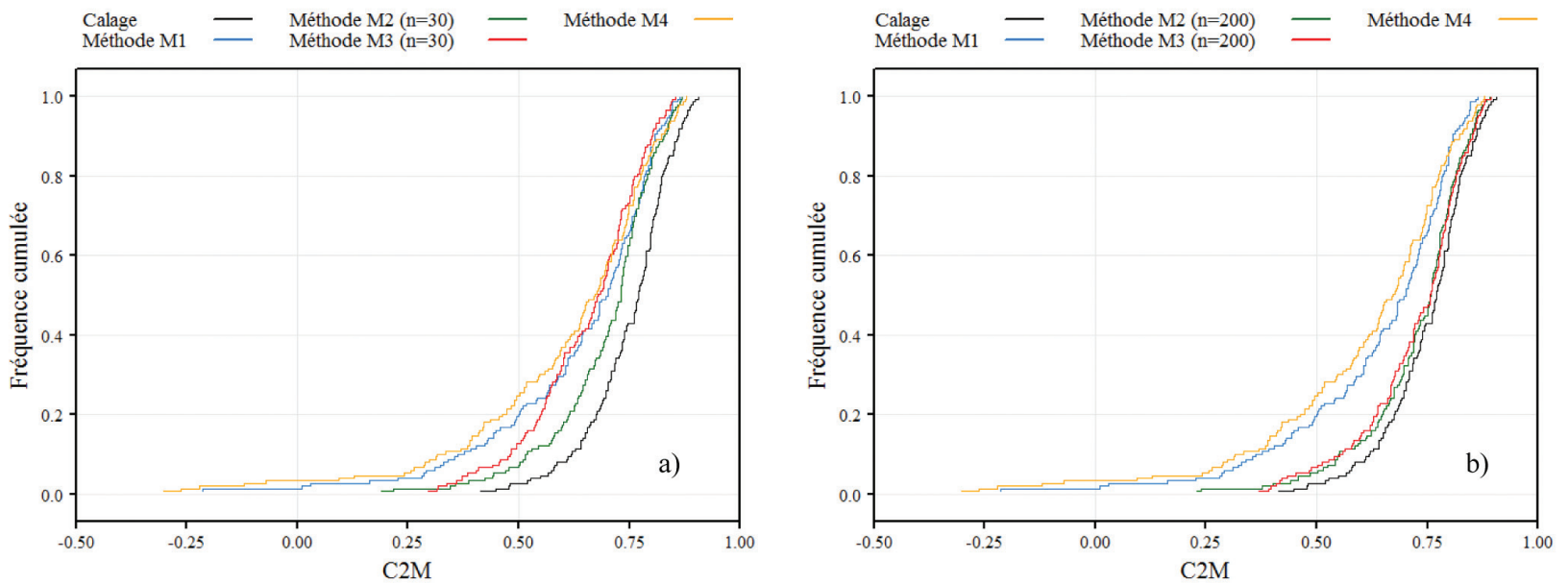

Figure 5 : Comparaison des performances des quatre méthodes de reconstitution du débit moyen journalier (149 stations hydrométriques) sur la période 1990-2009. Les courbes de fréquences cumulées des méthodes M2 et M3 sont présentées avec un nombre $n$ de débits ponctuels de 30 (a) et de 200 (b).

grandeur entre les distributions, en général plus étendue pour les débits instantanés. Les valeurs de Q10 (bas débits) calculés sur 30 débits ponctuels journaliers sont très majoritairement supérieures à celles calculées sur 200 valeurs.

C'est en général l'inverse qui se produit avec les valeurs de Q90 (forts débits). La gamme de débits est donc plus réduite pour l'échantillon de 30 débits ponctuels que pour celui de 200 débits ponctuels. Par ailleurs, en moyenne et selon nos hypothèses de travail, les performances du modèle GR4J sont meilleures en utilisant la méthode M3 plutôt que la méthode M1 sous réserve de caler le modèle au point-cible avec au moins 30 débits ponctuels ( 0.65 pour M3 vs 0.64 pour M1). Cet écart d' $1 / 100^{\mathrm{e}}$ de point C2M n'est cependant pas significatif du point de vue statistique (voir III.3).

\section{III.3. Efficacité comparée des méthodes de reconstitution du débit moyen journalier}

Les courbes de fréquence cumulées ( $\mathrm{CFC}$ ) du critère $\mathrm{C} 2 \mathrm{M}$ des quatre méthodes de reconstitution sont représentées sur la Figure 4. La CFC du critère $\mathrm{C} 2 \mathrm{M}$ en calage sert de référence : la valeur moyenne du critère $\mathrm{C} 2 \mathrm{M}$ vaut 0.75 , la valeur minimale, 0.42 . Notons que d'après le test de sensibilité réalisé par Mathevet (2005), qui tient compte de la valeur de l'écart-type des valeurs de $\mathrm{C} 2 \mathrm{M}$ et du nombre de stations, l'écart de performances entre les méthodes de reconstitution peut être considéré dans notre cas comme significatif s'il est supérieur à 0.05 .

Les méthodes M2 et M3 sont celles dont le niveau de performance est le plus proche de celui du modèle pluie-débit en calage, a fortiori lorsque $n$ est grand (Figure $4 \mathrm{~b}$ ). Le modèle hybride (méthode M2) possède le meilleur niveau de performance quelle que soit la densité de l'information hydrométrique ponctuelle disponible (Tableau 2). En revanche les performances des méthodes M1 et M4 sont assez similaires. Le fait de ne prendre en compte qu'un seul voisin (méthode M4) a pour conséquence d'augmenter l'écart-type du critère $\mathrm{C} 2 \mathrm{M}$.

Les résultats obtenus démontrent que la prise en compte d'une information hydrométrique ponctuelle, même 
Tableau 2: Statistique du critère C2M pour les quatre méthodes de reconstitution et en calage aux stations (période 1990-2009).

\begin{tabular}{|l|c|c|c|c|c|c|c|}
\hline $\begin{array}{c}\text { Statistique du critère } \\
\text { C2M }\end{array}$ & Calage & Méthode 1 & $\begin{array}{c}\text { Méthode 2 } \\
(\mathbf{n}=\mathbf{3 0})\end{array}$ & $\begin{array}{c}\text { Méthode 2 } \\
(\mathbf{n}=\mathbf{2 0 0})\end{array}$ & $\begin{array}{c}\text { Méthode 3 } \\
(\mathbf{n}=\mathbf{3 0})\end{array}$ & $\begin{array}{c}\text { Méthode 3 } \\
(\mathbf{n = 2 0 0})\end{array}$ & Méthode 4 \\
\hline Moyenne & 0.75 & 0.64 & 0.70 & 0.72 & 0.65 & 0.72 & 0.61 \\
\hline Médiane & 0.77 & 0.70 & 0.73 & 0.76 & 0.68 & 0.76 & 0.67 \\
\hline Ecart-type & 0.097 & 0.197 & 0.124 & 0.120 & 0.129 & 0.119 & 0.225 \\
\hline
\end{tabular}

parcimonieuse, permet d'améliorer significativement la reconstitution des débits journaliers au point-cible. Plusieurs éléments méthodologiques limitent néanmoins la portée opérationnelle de ces résultats :

- La performance des méthodes de reconstitution est dépendante de la paramétrisation des quatre méthodes de reconstitution, du modèle hydrologique implémenté et du critère de performance retenu $(\mathrm{C} 2 \mathrm{M})$. Il conviendrait d'évaluer le potentiel de généralisation de la méthode M2 en recourant à d'autres modèles hydrologiques et en changeant le critère de performance pour l'appliquer plutôt aux basses eaux ou aux hautes eaux selon l'objectif poursuivi.

- La prise en compte parmi les bassins-donneurs, d'un donneur ayant un vecteur de paramètres atypique (traduisant un problème d'hydrométrie, d'influence d'anthropique ou une singularité naturelle) aura pour conséquence de détériorer la reconstitution des chroniques issues des approches M1, M2 et M4 et de produire des erreurs importantes aux points-cibles. Cet inconvénient pose la question du « nettoyage » préalable de l'échantillon de bassins-donneurs. Le calcul d'un indice de discordance (rapport entre le C2M du donneur au point-cible et le $\mathrm{C} 2 \mathrm{M}$ en calage au point-cible) devrait permettre d'identifier les bassins-donneurs présentant une forte discordance avec le point-cible et qu'il conviendrait d'éliminer de l'échantillon de référence puis de remplacer par d'autres bassins-donneurs lors du processus de reconstitution du débit.

- Le tirage aléatoire des débits ponctuels a été mis en œuvre selon une loi uniforme sans critère de saisonnalité. Or les tests menés par Rojas-Serna (2005) ont montré qu'il était plus efficace de concentrer les mesures des débits ponctuels sur la période des hautes eaux, cela permettant en particulier de mieux estimer les paramètres contrôlant la dynamique du modèle GR4J en hautes eaux.

- Les débits ponctuels ont été tirés aléatoirement dans les chroniques de débits moyens journaliers. Or, en hydrométrie ponctuelle, le débit mesuré est un débit instantané. Selon la dynamique de l'écoulement au moment de la mesure et $\mathrm{du}$ caractère rapide ou lent de la réponse hydrologique du bassin, le débit instantané peut être représentatif ou contraire très différent du débit moyen journalier. Nos conclusions relatives au niveau de performance des méthodes testées sont donc surtout valables pour des bassins d'une certaine taille, plutôt pondérés et non pour des petits bassins fortement réactifs.

- La reconstitution des chroniques de débit aux stations des RCS contrôlant de petits bassins de quelques $\mathrm{km}^{2}$ voire quelques dizaines de $\mathrm{km}^{2}$ supposerait idéalement de recourir à un modèle pluie-débit global au pas de temps horaire, plus apte à reproduire la transformation de la pluie en débit et la variabilité temporelle de ce dernier à cette échelle spatiale. Les travaux de Mathevet (2005) permettent d'entrevoir une descente d'échelle temporelle à " moindre coût » dans la mesure où la structure du modèle GR4J ne serait pas plus complexe au pas de temps horaire qu'au pas de temps journalier et qu'une prédétermination robuste des paramètres du modèle horaire est possible à partir de son seul calage au pas de temps journalier. La mise en œuvre du modèle horaire suppose néanmoins de disposer de données d'entrée au même pas de temps. Or celles-ci sont plus parcellaires et plus récentes (séries courtes) que les données journalières.

\section{CONCLUSIONS}

La connaissance quantitative même fragmentaire, du débit en rivière, s'avère riche d'informations pour de nombreux utilisateurs. La reconstitution des chroniques continues de débits journaliers par simulation pluie-débit en l'absence de mesure directe et continue est une réponse pertinente à l'absence partielle ou totale de données hydrologiques en un point du réseau hydrographique. Cette approche, dans sa déclinaison à deux voisinages (géographique et hydrologique), fournit un niveau d'estimation satisfaisant du débit journalier aux stations hydrométriques à régime non influencé de la région étudiée. Elle est donc potentiellement transposable en tout point du réseau hydrographique jaugé ponctuellement ou temporairement et définissant une taille de bassin supérieure à quelques $\mathrm{km}^{2}$. Le niveau de performance de la simulation pluie-débit est optimal lorsqu'elle s'appuie sur des stations hydrométriques pérennes à longues chroniques ( $>10$ ans) et une hydrométrie ponctuelle parcimonieuse au point-cible (au moins 30 débits ponctuels).

Ce constat milite en faveur de la pérennisation de réseaux de stations hydrométriques patrimoniales et de la mise en œuvre régulière de campagnes de jaugeages volants, épisodiques, sur des bassins non jaugés, s'ajoutant aux campagnes de jaugeages aux stations destinés à établir leur courbe de tarage. Aux stations des RCS, il conviendrait a minima, de définir une stratégie de mesure quantitative de l'écoulement en rivière scrutant toute sa variabilité temporelle (hautes, moyennes, basses eaux) et tenant compte du coût financier des mesures et des contraintes logistiques. L'idéal serait de tendre vers l'implantation systématique de stations de jaugeages volantes (dispositif limnimétrique « léger », peu coûteux et facilement démontable) afin de disposer de quelques mois de chroniques de débits continues (débits auto-corrélés) qui pourraient ensuite être étendues par simulation pluie-débit éventuellement couplée à une simulation débit-débit.

Sur le plan théorique, les résultats préliminaires présentés dans cet article pourraient être poursuivis dans deux directions complémentaires : d'une part l'estimation de l'incertitude sur le débit reconstitué et d'autre part la prise en compte de la dépendance hydraulique entre stations hydrométriques contrôlant des bassins emboîtés. Cette prise en compte pourrait par exemple se traduire par une pondération différenciée entre bassins-donneurs selon leur degré de connectivité hydraulique avec le point-cible. 


\section{REMERCIEMENTS}

Météo-France (accès aux données SAFRAN), la DREAL Lorraine (accès banque HYDRO, BAREME), l'AERM (financement de l'étude et accès au SIERM), le German Federal Waterways and Shipping Administration ainsi que le German Federal Institute of Hydrology (BfG) (accès aux données allemandes), le Service public de Wallonie, Direction générale opérationnelle Mobilité et Voies hydrauliques, Direction de la Gestion hydrologique intégrée, Service d'Etudes Hydrologiques (SETHY) pour les données débitmétriques belges, mises à disposition gratuitement. Les auteurs souhaitent également remercier C. Perrin, V. Andréassian (IRSTEA Antony) et E. Leblois (IRSTEA Lyon) qui ont assuré l'encadrement de J. Plasse lors de son stage au sein de leur établissement ainsi que les trois relecteurs pour leurs commentaires constructifs sur la version initiale de cet article.

\section{REFERENCES}

Byrd R. H., Lu P., Nocedal J., Zhu C. (1995) - A limited memory algorithm for bound constrained optimization. SIAM. Journal on Scientific Computing. 16 : 1190-1208

MatheVet T. (2005) - Quels modèles pluie-débit globaux pour le pas de temps horaire? Développement empirique et comparaison de modèles sur un large échantillon de bassins versants. Thèse de doctorat, ENGREF (Paris), Cemagref (Antony), France. 463 p.

Mathevet T., Michel C., Andreassian V., Perrin C. (2006) A bounded version of the Nash-Sutcliffe criterion for better model assessment on large sets of basins. IAHS Red Books Series. 307 211-219

Oudin L., V. Andreassian C. Perrin C. Michel Et N. Le Moine (2008) - Spatial proximity, physical similarity and ungaged catchments: confrontation on 913 French catchments. doi03410.01029/02007WR006240. Water Resources Research. 44 : W03413

Perrin C., Michel C., Andreassian V. (2003) - Improvement of a parsimonious model for streamflow simulation. Journal of Hydrology. 279 : 275-289

Perrin C., Oudin L., Andréassian V., Rojas-Serna C., Michel C., Mathevet T. (2007) — Impact of limited streamflow knowledge on the efficiency and the parameters of rainfall-runoff models. Hydrological Sciences Journal. 52(1) : 131-151

Quintana-Segui P., Le Moigne P., Durand Y., Martin E., Habets F., Baillon M., Canellas C., Franchisteguy L., Morel S. (2008) - Analysis of near-surface atmospheric variables: Validation of the SAFRAN analysis over France. Journal of Applied Meteorology and Climatology. 47 : 92-107

ROJAS-SERNA C. (2005) - Quelle connaissance hydrométrique minimale pour définir les paramètres d'un modèle pluie-débit ? Thèse de doctorat, ENGREF (Paris), Cemagref (Antony), France. $320 \mathrm{p}$

Rojas-Serna C., Perrin C., Andréassian V., Oudin L. How should a rainfall-runoff model be parameterized in an almost ungauged catchment? A methodology tested on 705 catchments. Soumis à Water Resources Research

Seibert, J., Beven, K. J. (2009) - Gauging the ungauged basin: how many discharge measurements are needed? doi10.5194/ hess-13-883-2009. Hydrology and Earth System Sciences. 13 : $883-892$ 